\title{
Decay of low-angle tilt boundaries in deformed nanocrystalline materials
}

\author{
S V Bobylev, M Yu Gutkin and I A Ovid'ko \\ Institute of Problems of Mechanical Engineering, Russian Academy of Sciences, Bolshoj 61, \\ Vas. Ostrov, St Petersburg 199178, Russia \\ E-mail: ovidko@def.ipme.ru
}

Received 1 October 2003

Published 19 December 2003

Online at stacks.iop.org/JPhysD/37/269 (DOI: 10.1088/0022-3727/37/2/016)

\begin{abstract}
A theoretical model is suggested, which describes a decay of low-angle grain boundaries in deformed nanocrystalline materials (NCMs). In the framework of the model, lattice dislocations that form a low-angle boundary are under the action of the forces owing to external (applied) and internal stresses. The balance of the forces causes the critical shear stress at which a low-angle boundary decays. Such decay processes result in the formation of high-density ensembles of mobile lattice dislocations that are capable of inducing plastic flow localization (shear banding) in mechanically loaded NCMs.
\end{abstract}

\section{Introduction}

Nanocrystalline materials (NCMs) exhibit outstanding deformation behaviour whose fundamental mechanisms are under intensive discussion (see, e.g., [1-15]). Such materials are often extremely hard and brittle [16], but some nanocrystalline metals, ceramics and alloys exhibit even superplasticity [17-21]. The ductility of an NCM is often controlled by its ability to suppress plastic flow localization in shear bands, nuclei of the necking [22]. This has generated interest in the identification of deformation mechanism(s) acting in shear bands in NCMs, which potentially will allow one to influence the processes of plastic flow localization. In general, plastic and superplastic deformation mechanisms in NCMs are supposed to be lattice dislocation slip $[1,2]$, grain boundary (GB) sliding [10-14], GB diffusional creep [3-5], triple junction diffusional creep [6], twinning [15] and rotational deformation mode [7-10], which compete with each other. In this paper, we will focus our consideration on ductile NCMs with comparatively large grains with size $d \geqslant 30 \mathrm{~nm}$, in which the lattice dislocation slip is dominant. The action of conventional lattice dislocation sources such as Frank-Read sources is modified and sometimes suppressed in such materials due to nanoscale and interface effects [23]. In these circumstances, GBs whose volume fraction is very high in NCMs can serve as effective sources of lattice dislocations $[9,10]$, strongly affecting plastic flow. In previous theoretical models $[9,10]$ of emission of lattice dislocations by high-angle GBs in nano- and fine-grained materials, the intensity of the emission events is assumed to be controlled by movement and transformations of GB dislocations and disclinations. These processes are too slow to cause the formation of high-density ensembles of mobile lattice dislocations that would carry large plastic strains in shear bands. At the same time, NCMs contain low-angle boundaries consisting of lattice dislocations [24]. Such low-angle boundaries undergo structural transformations under the action of internal stresses in coarse-grained polycrystalline materials (see [25-27] and references therein), in which case it is natural to think that low-angle boundaries can undergo dramatic transformations under the action of applied stresses in mechanically loaded NCMs, too. The main aim of this paper is to suggest a theoretical model that describes decays of low-angle tilt boundaries under the shear stress action as processes that produce high-density ensembles of mobile lattice dislocations in local regions of NCMs.

\section{Model}

Let us consider a model low-angle tilt boundary terminated at triple junctions of GBs in a nanocrystalline sample (figure $1(a)$ ). The low-angle boundary in its initial state in the absence of mechanical load is represented as a straight wall of periodically arranged edge dislocations, each having Burgers vector $\boldsymbol{b}$. The low-angle boundary is characterized by the tilt misorientation angle $\theta$ being in the Frank relationship $(\sin (\theta / 2)=b / 2 h[25])$ with parameters (period $h$ and Burgers vector magnitude $b$ ) of the dislocation arrangement in the 
(a)

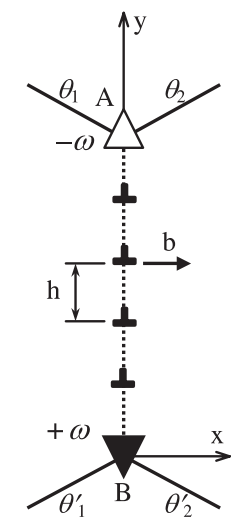

(b)

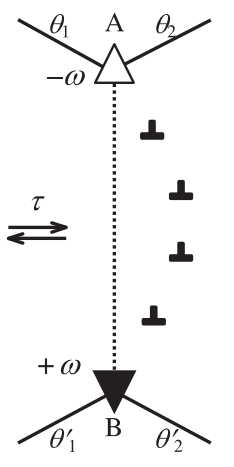

(c)

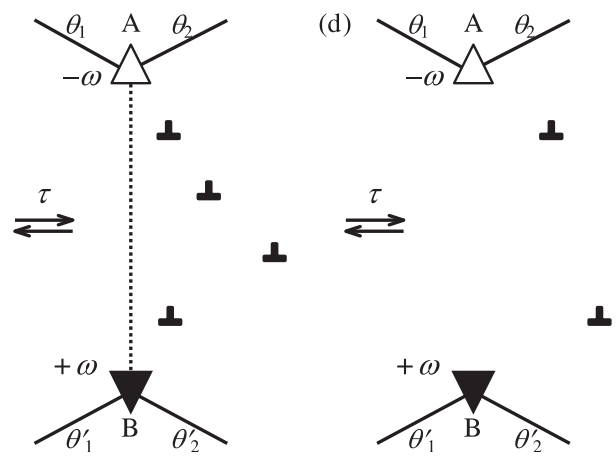

Figure 1. Decay of low-angle tilt boundary with the misorientation angle $\theta$ represented as a periodic dislocation wall terminated at triple junctions $\mathrm{A}$ and $\mathrm{B}$ (parameters $\theta_{1}, \theta_{2}, \theta_{1}^{\prime}, \theta_{2}^{\prime}$ are misorientation angles of adjacent boundaries). (a) Dislocation wall in its initial state (in the absence of mechanical load). (b) Shear stress $\tau$ causes blowing of the dislocation wall. (c) One of the dislocations releases from the dislocation wall. $(d)$ Dislocation wall completely decays resulting in the formation of a dipole of uncompensated disclinations with strengths $-\omega$ and $+\omega$ at triple junctions $\mathrm{A}$ and $\mathrm{B}$, respectively.

boundary. Also, for definiteness, the tilt misorientation $\theta$ is assumed to be in compensating relationships, $\theta+\theta_{1}+\theta_{2}=0$ and $-\theta+\theta_{1}^{\prime}+\theta_{2}^{\prime}=0$, with the tilt misorientation parameters, $\left(\theta_{1}, \theta_{2}\right)$ and $\left(\theta_{1}^{\prime}, \theta_{2}^{\prime}\right)$, of the GBs adjacent, respectively, to the upper and bottom triple junctions shown in figure 1(a). In other words, the finite dislocation wall in its initial state (figure $1(a)$ ) is terminated by disclinations that completely compensate for the disclination defects (shown as triangles in figure 1) at the upper and lower junctions of its adjacent GBs characterized by the tilt misorientation parameters, $\left(\theta_{1}, \theta_{2}\right)$ and $\left(\theta_{1}^{\prime}, \theta_{2}^{\prime}\right)$, respectively.

The action of a shear stress $\tau$ on the edge dislocations composing the low-angle tilt boundary in a mechanically loaded nanocrystalline sample causes displacements of these dislocations (figure $1(b)$ ) from their initial positions shown in figure $1(a)$. At some critical value $\tau_{\mathrm{c}}$ of the shear stress, one of the edge lattice dislocations composing the low-angle boundary releases and starts moving far from the initial boundary plane (figure $1(c)$ ). As is evident (and proved by further analysis in this paper), the release of the first dislocation from the low-angle boundary (figure $1(c)$ ) is immediately followed by decay of the boundary (dislocation wall) as a whole (figure $1(d)$ ). As a result, a group of lattice dislocations

released from the decayed low-angle boundary move, causing local plastic deformation and the formation of an elongated grain. Following experimental data [22, 28], such elongated grains are characteristic structural elements of shear bands in nanocrystalline Fe.

Notice that the decay of the low-angle boundary removes it from the triple junctions, which thereafter transform into uncompensated double junctions (figure $1(d)$ ). The upper and lower junctions become uncompensated stress sources of the disclination type, characterized by disclination strength $-\omega=-\theta$ and $\omega=\theta$, respectively. The stress field of the disclination dipole serves as the hampering force for movement (release) of the lattice dislocations from the low-angle tilt boundary and its decay. It is taken into account in our calculations of the critical shear stress $\tau_{\mathrm{c}}$ (at which the decay occurs) in the rest of this paper.

\section{Results and discussion}

Let us calculate the critical shear stress $\tau_{\mathrm{c}}$ by methods of two-dimensional dislocation dynamics. We have used the two-dimensional approximation just to catch the principal peculiarities of the decay process and roughly estimate the $\tau_{\mathrm{c}}$. A more accurate three-dimensional simulation is much more cumbersome and is now under preparation. We expect that the results of both the two- and three-dimensional approaches would give similar results in the case when the dislocation displacements are small compared to the length of the boundary.

Each dislocation in the low-angle boundary in a mechanically loaded NCM (figure $1(b)$ ) is under the combined action of the forces caused by the shear stress, other dislocations belonging to the boundary, and the disclination dipole. Let us calculate these forces and write the corresponding equations for dislocation movement. In doing so, we assume that dislocations can move along one slip plane (along the $x$-axis in the coordinate system shown in figure $1(a)$ ), in which case only the $x$-projections of the forces play the role. The solution of the system of equations, describing one-dimensional movement of dislocations, will be expressed as dependences $x_{i}(t)$, where $x_{i}$ is the coordinate of the $i$ th dislocation $(i=1, \ldots, N)$, and $t$ is time.

In the framework of the approach discussed, the force acting on the $i$ th dislocation belonging to the low-angle boundary may be written as follows:

$$
\begin{aligned}
F_{i}= & b \tau+D b^{2} \sum_{\substack{k=1 \\
k \neq i}}^{N} \frac{\left(x_{i}-x_{k}\right)\left\{\left(x_{i}-x_{k}\right)^{2}-\left(y_{i}-y_{k}\right)^{2}\right\}}{\left\{\left(x_{i}-x_{k}\right)^{2}+\left(y_{i}-y_{k}\right)^{2}\right\}^{2}} \\
& -D b \omega\left(\frac{x_{i} y_{i}}{x_{i}^{2}+y_{i}^{2}}-\frac{x_{i}\left(y_{i}-d\right)}{x_{i}^{2}+\left(y_{i}-d\right)^{2}}\right),
\end{aligned}
$$

where $D=G /[2 \pi(1-v)], G$ is the shear modulus, $v$ is the Poisson ratio, $x_{i}$ and $y_{i}=i h$ are the coordinates of the $i$ th dislocation, and $d$ denotes the tilt boundary length (the distance between the triple junction disclinations that form the dipole (see figure 1)). The first term of formula (1) describes the force due to the shear stress $\tau$, the second term describes the force of interaction with the other dislocations of the boundary [29], and the third describes the force of interaction with the disclination dipole [30]. 
With the forces given by formula (1), equations for movement of the dislocations composing the low-angle tilt boundary (figure $1(a)$ ) read

$$
m \frac{\mathrm{d}^{2} x_{i}}{\mathrm{~d} t^{2}}+\beta \frac{\mathrm{d} x_{i}}{\mathrm{~d} t}=F_{i}, \quad i=1,2, \ldots, N .
$$

First derivatives $\mathrm{d} x_{i} / \mathrm{d} t$ in these equations take into account the dislocation movement friction (associated with the dynamic retardation of the crystalline lattice to dislocation movement), and $\beta$ is the viscosity coefficient. The dislocation mass $m$ is given by the standard approximation [31], $m=\rho b^{2} / 2$, where $\rho$ is the material density.

To solve numerically the system of equation (2), we have used the conventional software Mathematica 4. The following characteristic values of parameters have been chosen for the exemplary case of nanocrystalline $\mathrm{Fe}$ (whose deformation behaviour was experimentally studied in [22,28]): $G=$ $82 \mathrm{GPa}, v=0.29$, lattice parameter $a=0.287 \mathrm{~nm}, b=$ $\frac{1}{2} a\langle 111\rangle=0.25 \mathrm{~nm}, \rho=7800 \mathrm{~kg} \mathrm{~m}^{-3}$, dislocation mass (per unit length of dislocation line) $m=2.4 \times 10^{-16} \mathrm{~kg} \mathrm{~m}^{-1}$, and $\beta \approx 5 \times 10^{-5} \mathrm{Pas}$ [31]. For these values of parameters, we have considered the behaviour of a low-angle tilt boundary composed of $N=10$ dislocations and characterized by tilt misorientation angle $\theta=0.1\left(\approx 5.7^{\circ}\right)$, in which case $\omega=0.1$ and $h=10 b$.

Some typical plots $x_{5}(t)$, for the fifth dislocation located at the boundary centre, have been calculated at different values of $\tau$ ranging from 0.5 to $1.6 \mathrm{GPa}$ (see figure 2). For our analysis, the fifth dislocation at the GB centre has been chosen, because the decay of a boundary is expected to begin at its centre where the effect of the disclination dipole is minimized. This expectation has been confirmed by our calculations. For relatively low stress values (here $\leqslant 1.57 \mathrm{GPa}$ ), the fifth dislocation makes some oscillations and is finally stabilized at some equilibrium position, which is shifted further with increasing stress value (curves $1-5$ in figure 2 ). When the stress magnitude becomes larger than a critical value $\tau_{\mathrm{c}}$ (here $\left.\tau_{\mathrm{c}}=1.58 \mathrm{GPa}\right)$, the dislocation moves far away from its initial position (curves 6 and 7 in figure 2).

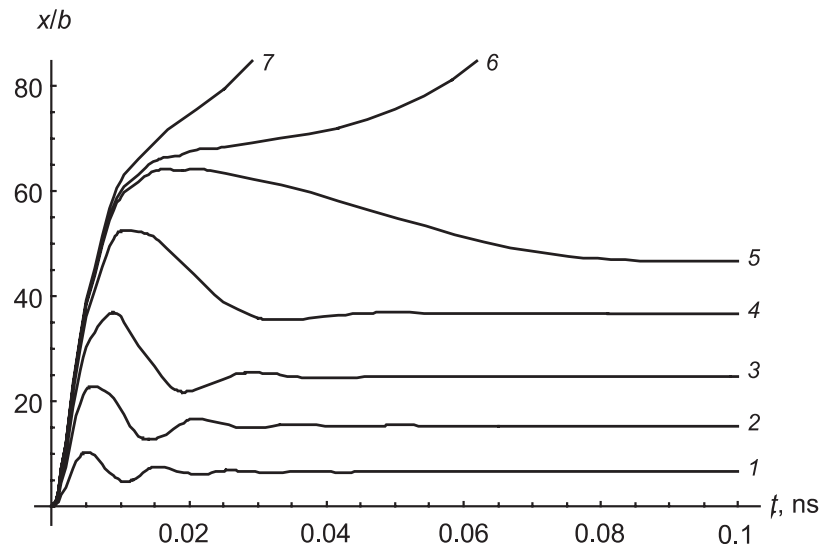

Figure 2. Plots $x_{5}(t)$ for the fifth dislocation, one of two central dislocations of a low-angle tilt boundary consisting of $N=10$ edge dislocations and having the misorientation angle $\theta=0.1 \approx 5.7^{\circ}$, for different values of the shear stress $\tau=0.5$ (curve 1), 1 (2), 1.3 (3),

$1.5(4), 1.57(5), 1.58(6)$ and $1.6 \mathrm{GPa}(7)$.
As noted earlier, the decay of the boundary begins at its centre when the fifth (central) dislocation is released from the boundary (figure 1(c)). Following our calculations, immediately after the central dislocation has moved far enough from the initial boundary plane (figure $1(c)$ ), the boundary dramatically decays as a whole (figure $1(d)$ ). That is, all the dislocations composing the boundary move far from its plane during a very short time interval. In these circumstances, in order to calculate critical parameters at which the low-angle boundary decays as a whole, it is sufficient to characterize the behaviour of only one (central) dislocation.

In this manner, we have analysed stability and decay of low-angle tilt boundaries with different parameters. This has allowed us to reveal critical values of the shear stress $\tau_{\mathrm{c}}$ at which the decay (figure 1) of low-angle tilt boundaries with different characteristics occurs. The dependence of $\tau_{\mathrm{c}}$ on boundary misorientation $\theta$ is shown in figure 3 , for $N=5$, 10 and 20. As follows from figure $3, \tau$ grows in a tentatively linear way with rising $\theta$. It is indicative of the crucial contribution of the boundary misorientation and thereby the strength of the disclination dipole to the stability of lowangle tilt boundaries under the shear stress action. Curves corresponding to different values of parameter $N$ at a constant value of $\theta$ are very close (see figure 3 ). This means that the dependence of $\tau_{\mathrm{c}}$ on the number $N$ of dislocations and thereby the boundary length $d(=N h)$ at a constant value of $\theta$ is very weak. In this context, in particular, very short GBs in very small grains and comparatively long GBs in large grains decay at close values of the critical stress $\tau_{\mathrm{c}}$, if they have the same misorientation parameters. At the same time, the action of Frank-Read sources of lattice dislocations is suppressed in very small grains of NCMs in which only the decay of low-angle boundaries provides the effective generation of mobile lattice dislocations. In this event, $\tau_{\mathrm{c}}$ represents a very important characteristic of plastic flow in NCMs. In contrast, in conventional coarse-grained materials, where Frank-Read sources are activated at shear stresses lower than $\tau_{\mathrm{c}}$, the decay of low-angle boundaries does not play any essential role in plastic deformation processes.

The decay of a low-angle tilt boundary in a mechanically loaded nanocrystalline sample results in the formation of moving lattice dislocations that carry local plastic deformation and come to the neighbouring GBs (figure $1(d)$ ). The lattice

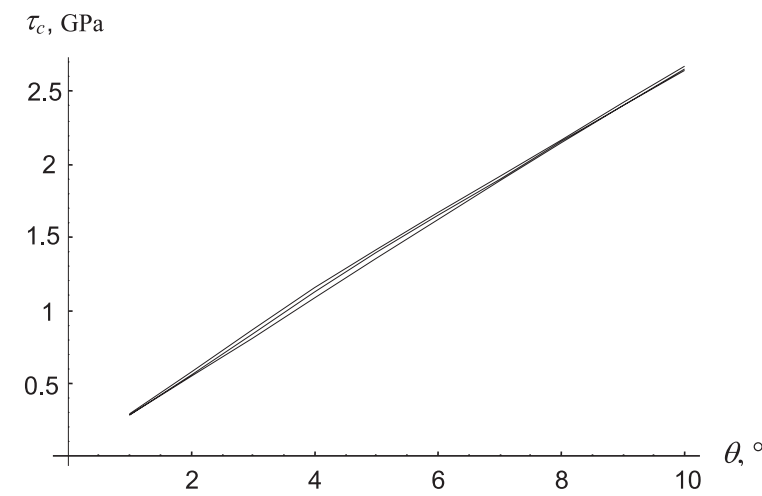

Figure 3. Dependence of the critical shear stress $\tau_{\mathrm{c}}$ on the misorientation angle $\theta$ of a tilt boundary consisting of $N=5,10$ and 20 edge dislocations (from top to bottom). The critical stress $\tau_{c}$ characterizes the decay of the tilt boundary as a whole (see text). 
dislocations elastically interact with other lattice dislocations composing neighbouring low-angle GBs. This interaction is able to cause decays of the neighbouring low-angle GBs, accompanied by avalanche-like release of new mobile lattice dislocations.

\section{Conclusions}

Thus, the decay of a low-angle boundary in a mechanically loaded nanocrystalline solid gives rise to local plastic deformation in the grain where the low-angle boundary decay has occurred and in its neighbouring grains. In this context, the shear-stress-induced decay of a low-angle tilt boundary can trigger the formation of a shear band (thin, sheet-like region where high plastic strain is localized) in the nanocrystalline solid. In this event, the critical shear stress $\tau_{\mathrm{c}}$ characterizes the initial stage of local plastic deformation occurring via generation and development of shear bands. According to calculations given in this paper (see figure 3 ), the shear stress $\tau_{\mathrm{c}}$ ranges from $0.5 \mathrm{GPa}$ (for $\theta \approx 2^{\circ}$ ) to $2.5 \mathrm{GPa}$ (for $\theta \approx 10^{\circ}$ ), which characterizes the decay of low-angle tilt boundaries with different misorientation parameters in nanocrystalline Fe. Its mean value $\left\langle\tau_{\mathrm{c}}\right\rangle=1.5 \mathrm{GPa}$ coincides with the experimentally measured [28] value of the shear stress at which shear bands with elongated grain structure are formed in nanocrystalline $\mathrm{Fe}$.

\section{Acknowledgments}

This work was supported, in part, by the Office of US Naval Research (grant N00014-01-1-1020), the Russian Fund of Basic Research (grant 01-02-16853), Russian State Research Program on Solid-State Nanostructures, Russian Academy of Sciences Program 'Structural Mechanics of Materials and Constructions', St Petersburg Scientific Center, and 'Integration' Program (grant B0026).

\section{References}

[1] Pande C S, Masumura R A and Armstrong R W 1993 Nanostruct. Mater. 2 323-31

[2] Malygin G A 1995 Phys. Solid State 37 1248-53

[3] Masumura R A, Hazzledine P M and Pande C S 1998 Acta Mater. 46 4527-34
[4] Kim H S, Estrin Y and Bush M B 2000 Acta Mater. 48 493-504

[5] Yamakov V, Wolf D, Phillpot S R and Gleiter H 2002 Acta Mater. 50 61-73

[6] Fedorov A A, Gutkin M Yu and Ovid'ko I A 2002 Scr. Mater 47 51-5

[7] Murayama M, Howe J M, Hidaka H and Takaki S 2002 Science 295 2433-5

[8] Ovid'ko I A 2002 Science 2952386

[9] Gutkin M Yu, Kolesnikova A L, Ovid'ko I A and Skiba N V 2002 Phil. Mag. Lett. 82 651-7

[10] Gutkin M Yu, Ovid'ko I A and Skiba N V 2003 Acta Mater. 51 4059-71

[11] Hahn H, Mondal P and Padmanabhan K A 1997 Nanostruct. Mater. 9 603-6

[12] Hahn H and Padmanabhan K A 1997 Phil. Mag. B 76 559-71

[13] Konstantinidis D A and Aifantis E C 1998 Nanostruct. Mater. $101111-8$

[14] Fedorov A A, Gutkin M Yu and Ovid'ko I A 2003 Acta Mater. $51887-98$

[15] Chen M, Ma E, Hemker K J, Sheng H, Wang Y and Cheng X 2003 Science 300 1275-7

[16] Mohamed F A and Li Y 2001 Mater. Sci. Eng. A 298 1-15

[17] McFadden S X, Misra R S, Valiev R Z, Zhilyaev A P and Mukherjee A K 1999 Nature 398 684-6

[18] Valiev R Z, Song C, McFadden S X, Mukherjee A K and Mishra R S 2001 Phil. Mag. A 81 25-36

[19] Mukherjee A K 2002 Mater. Sci. Eng. A 322 1-22

[20] Valiev R Z, Alexandrov I V, Zhu Y T and Lowe T C 2002 J. Mater. Res. 17 5-8

[21] Mukherjee A K 2002 Creep Deformation: Fundamentals and Applications ed R S Mishra et al (Warrendale: TMS) pp 3-19

[22] Jia D, Ramesh K T and Ma E 2003 Acta Mater. 51 3495-509

[23] Weertman J R and Sanders P G 1994 Solid State Phenom. 35-36 249-62

[24] Zghal S, Hytch M J, Chevalier J-P, Twesten R, Wu F and Bellon P 2002 Acta Mater. 504695

[25] Sutton A P and Balluffi R W 1995 Interfaces in Crystalline Materials (Oxford: Clarendon) pp 70-96

[26] Gutkin M Yu and Ovid'ko I A 2001 Phys. Rev. B 6364515

[27] Bobylev S V and Ovid'ko I A 2003 Phys. Rev. B 67132506

[28] Wei Q, Jia D, Ramesh K T and Ma E 2002 Appl. Phys. Lett. 81 1240-2

[29] Hirth J P and Lothe J 1982 Theory of Dislocations (New York: Wiley)

[30] Romanov A E and Vladimirov V I 1992 Dislocations in Solids vol 9, ed F R N Nabarro (Amsterdam: North-Holland) pp 191-402

[31] Kocks U F, Argon A S and Ashby M F 1975 Prog. Mater. Sci. $191-288$ 